\title{
Effects of loss aversion on neural responses to loss outcomes: an event- related potential study
}

Katerina Kokmotou ${ }^{1,2}$, Stephanie Cook ${ }^{1}$, Yuxin Xie ${ }^{3}$, Hazel Wright ${ }^{1}$, Vicente Soto ${ }^{1}$, Nicholas Fallon ${ }^{1}$, Timo Giesbrecht ${ }^{4}$, Athanasios Pantelous ${ }^{2,5}$, Andrej Stancak ${ }^{1,2}$

${ }^{1}$ Department of Psychological Sciences, University of Liverpool, Liverpool, UK.

${ }^{2}$ Institute for Risk and Uncertainty, University of Liverpool, Liverpool, UK.

${ }^{3}$ School of Securities and Futures, Southwestern University of Finance and Economics, China.

${ }^{4}$ Unilever, Research and Development, Port Sunlight, UK.

${ }^{5}$ Department of Mathematical Sciences, University of Liverpool, Liverpool, UK.

Corresponding author:

Dr. Andrej Stancak

Department of Psychological Sciences

University of Liverpool

Liverpool, L69 7ZA

United Kingdom

Email: a.stancak@liverpool.ac.uk

Phone: +44 1517946951 


\begin{abstract}
Loss aversion is the tendency to prefer avoiding losses over acquiring gains of the same amount. To shed light on the spatio-temporal processes underlying loss aversion, we analysed the associations between individual loss aversion and electrophysiological responses to loss and gain outcomes in a monetary gamble task. Electroencephalographic feedback-related negativity (FRN) was computed in 29 healthy participants as the difference in electrical potentials between losses and gains. Loss aversion was evaluated using non-linear parametric fitting of choices in a separate gamble task. Loss aversion correlated positively with FRN amplitude (233-263 ms) at electrodes covering the lower face. Feedback related potentials were modelled by five equivalent source dipoles. From these dipoles, stronger activity in a source located in the orbitofrontal cortex was associated with loss aversion. The results suggest that loss aversion implemented during risky decision making is related to a valuation process in the orbitofrontal cortex, which manifests during learning choice outcomes.
\end{abstract}




\section{Introduction}

Loss aversion is the tendency to prefer avoiding losses over acquiring gains of the same amount (Kahneman \& Tversky, 1979). Loss aversion affects a large range of economic behaviours, such as willingness to part with an object in one's possession (Kahneman, Knetsch, \& Thaler, 1990), relative sensitivity to price changes (Hardie, Johnson, \& Fader, 1993; Putler, 1992), decision making in a monetary gamble task (Sokol-Hessner et al., 2009; Takahashi et al., 2012; Tom, Fox, Trepel, \& Poldrack, 2007), or the style of playing golf (Pope \& Schweitzer, 2011).

In prospect theory of decision making (Kahneman \& Tversky, 1979), individual decisions are modelled by two functions, the probability weighting function and the utility function. Loss aversion, typically evaluated in tasks involving decision-making under risk (Barkley-Levenson, Van Leijenhorst, \& Galván, 2013; Canessa et al., 2013; Tom et al., 2007; Wright et al., 2012), is defined as a utility function that is steeper for losses than for gains of equal size. Similarly, losses are associated with greater autonomic (Sokol-Hessner et al., 2009; Stancak et al., 2015) and cerebral (Sokol-Hessner, Camerer, \& Phelps, 2013; Tom et al., 2007) responses in people with strong loss aversion compared to people with small loss aversion. Individual levels of loss aversion have been shown to negatively correlate with the presence of norepinephrine transporters in the thalamus (Takahashi et al., 2012). Further, a recent structural magnetic resonance imaging (MRI) study revealed a positive correlation between loss aversion and grey matter volume in amygdala, thalamus and striatum (Canessa et al., 2013). Together, the above results suggest that loss aversion may operate as a relatively stable feature during decision making (Glöckner \& Pachur, 2012), although loss aversion can also be modulated by the task or context (Schulreich, Gerhardt, \& Heekeren, 2016; SokolHessner et al., 2013; Stancak et al., 2015). 
A loss in a monetary gamble task is a negative feedback. A wealth of electrophysiological data suggests that presenting information about losses compared to gains is associated with a negative deflection in the electrocortical potential, which is superimposed on the subsequent, typically large positive P300 component (Nieuwenhuis, Yeung, Holroyd, Schurger, \& Cohen, 2004; Yeung, Holroyd, \& Cohen, 2005). This negative electrical potential, known as feedback-related negativity (FRN), occurs between 200 and 350 ms (Gehring \& Willoughby, 2002; Miltner, Braun, \& Coles, 1997; Nieuwenhuis, Holroyd, Mol, \& Coles, 2004; Walsh \& Anderson, 2012) and shows a characteristic scalp potential map with a spatial maximum in the fronto-central midline region of the scalp (Gehring \& Willoughby, 2002; Hajcak, Moser, Holroyd, \& Simons, 2006; Nieuwenhuis, Yeung, et al., 2004; Walsh \& Anderson, 2012; Yeung \& Sanfey, 2004). The cortical source of FRN has been located near or in the anterior cingulate cortex (Bellebaum \& Daum, 2008; Gehring \& Willoughby, 2002; Hewig et al., 2007; Miltner et al., 1997; Potts, Martin, Burton, \& Montague, 2006; Ruchsow, Grothe, Spitzer, \& Kiefer, 2002). However, the potential fields during the period of FRN appear to have a more complex topography with positive components occupying the bilateral temporal regions of the scalp, suggesting the possibility that multiple cortical sources might be involved (Gehring \& Willoughby, 2002). Indeed, several studies have identified additional brain regions contributing to the generation of FRN (for reviews see Hauser et al., 2014; Walsh \& Anderson, 2012), such as the posterior cingulate cortex (Badgaiyan \& Posner, 1998; Cohen \& Ranganath, 2007; Müller, Möller, Rodriguez-Fornells, \& Münte, 2005; Nieuwenhuis et al., 2005) and the striatum (Martin, Potts, Burton, \& Montague, 2009; Nieuwenhuis et al., 2005).

In the context of the present study, punishment sensitivity has been shown to be related to the amplitude of FRN (Santesso, Dzyundzyak, \& Segalowitz, 2011; Unger, Heintz, \& Kray, 2012). In studies exploring effects of framing, stronger FRN amplitudes were found in 
prospects framed negatively compared to those framed positively (Ma, Feng, $\mathrm{Xu}, \mathrm{Bian}, \&$ Tang, 2012; Yu \& Zhang, 2014). Further, a recent ERP study showed that loss aversion attenuated amplitudes of a posterior positive slow wave during decisions involving low conflict between competing options (Heeren, Markett, Montag, Gibbons, \& Reuter, 2016). These studies suggest the possibility of an association between FRN and loss aversion.

The purpose of the present study was to identify the cortical regions and time period when loss aversion modulates the cortical response to losses during the evaluation of choice outcomes. Although loss aversion affects decision making during the period of evaluation of expected utilities of individual prospects, previous studies also found processing of loss outcomes related to loss aversion (Sokol-Hessner et al., 2013; Sokol-Hessner et al., 2009; Stancak et al., 2015). Neural responses to expected (Knutson, Adams, Fong, \& Hommer, 2001) and actually perceived (Delgado, Nystrom, Fissell, Noll, \& Fiez, 2000; May et al., 2004) losses or gains are processed in an overlapping set of regions. Meta-analyses of fMRI studies typically point to ventral striatum, orbitofrontal and ventromedial prefrontal cortex as playing a central role in value-based decision making (Bartra, McGuire, \& Kable, 2013; Clithero \& Rangel, 2014). Therefore, we postulated that loss aversion will be associated with the electrophysiological responses to choice outcomes in one or more regions belonging to the brain valuation system (Bartra et al., 2013; Clithero \& Rangel, 2014; Lebreton, Jorge, Michel, Thirion, \& Pessiglione, 2009). To identify the brain regions involved in mediating the relationship between loss aversion and FRN, we applied source dipole analysis and analysed the associations between source dipole waveforms and loss aversion using correlation analysis. To differentiate the effects of sensitivity to losses from sensitivity to risk, a nonlinear parametric method was applied to model the individual choices using three parameters: loss aversion, curvature of the value function and choice sensitivity (Sokol-Hessner et al., 2013; Sokol-Hessner et al., 2009; Stancak et al., 2015). Although the primary focus of the 
present study was on loss aversion, the curvature of the value function was evaluated as well to check the potentially overlapping effects of these two preference parameters. Finally, choice sensitivity served as an estimation of participants' response consistency throughout the experiment.

\section{Methods}

\subsection{Participants}

A total of 31 participants (16 females) completed the study. Two participants were removed from subsequent analyses due to technical issues encountered during EEG recordings. Thus, the final sample included 29 participants (14 females), aged $22.5 \pm 3.6$ years (mean \pm SD), 4 left-handed. The experimental procedures were approved by the Research Ethics Committee of the University of Liverpool. All participants gave written informed consent in accordance with the Declaration of Helsinki.

\subsection{Procedure}

The experiment involved two different tasks. The first one was a monetary gamble task comprising 100 trials. Participants had to select between two prospects with one of them offering a sure zero outcome or sure non-zero gain and the other an uncertain gain or loss of variable amounts. This task was used to assess individual loss aversion levels. Next, participants completed an EEG experiment involving only uncertain monetary gambles followed by presentation of the outcome. The event-related potential analysis of the outcome period served to evaluate the individual FRN potentials. The purpose of the experiment was explained to participants, who were given instructions for the tasks at the beginning of the session. 


\subsection{Loss aversion task}

The initial monetary gamble task was adapted from previous studies (Sokol-Hessner et al., 2013; Sokol-Hessner et al., 2009; Tom et al., 2007), and in particular from Stancak et al. (2015). Participants received an initial endowment of $£ 20$ and were instructed to use it for gambling during the experiment. They were informed that $10 \%$ of the difference between their total gains and losses would be added to or subtracted from this $£ 20$ endowment and they would receive the remaining amount as a reimbursement for their participation.

The task consisted of a total of 100 trials. In 80 of those trials, participants decided between a gamble and an alternative sure zero outcome. Each gamble consisted of 8 possible gain amounts $(£ 1.0, £ 2.0, £ 3.0, £ 3.5, £ 4.5, £ 5.5, £ 5.0, £ 6.0)$ in combination with 10 possible losses. The losses were computed by multiplying each particular gain value with a coefficient from 0.2 to 2.0 in 0.2 steps in all possible permutations ( 8 gains $\times 10$ losses). Potential gains and losses were associated with equal probabilities (i.e., 50\%). In additional 20 trials, participants decided between a gain-only gamble and a sure non-zero outcome. Here, the gain-only gambles offered a $50 \%$ chance to win a certain gain amount or zero otherwise, whereas the sure alternative was a smaller gain. These 20 gambles were identical with those listed in Table 1 in our previous study (Stancak et al., 2015). Trials were presented in random order for each participant.

Participants were seated in front of a 19-inch CRT monitor, and rested their right hand on a computer mouse. The stimuli were presented using Cogent software 2000 (UCL, London, United Kingdom) for Matlab (Mathworks, Inc., USA). The trial structure is shown in Figure 1A. Each trial began with a fixation cross that stayed on the screen for 1 second. Subsequently, two possible choices were displayed on the screen for $4 \mathrm{~s}$. Half of the screen presented a gamble option (e.g. "you win $£ 3.0$, you lose $£ 3.0 ”)$ in yellow text on black background. Participants were informed that the outcome was always random (i.e., with $50 \%$ probability). 
The other half of the screen showed the value of a sure outcome (e.g., £0). They were instructed to choose between the two prospects by pressing the left or right mouse button according to the part of the screen they preferred. If the participant selected the risky gamble option, feedback about the outcome was shown for $1 \mathrm{~s}$ ("you won" or "you lost"). The duration of this initial gamble task was approximately 15 minutes.

\subsection{The FRN experiment}

After completing the loss aversion task, participants were connected to the EEG system. EEG was recorded continuously using a 129-channel Geodesics EGI System (Electrical Geodesics, Inc., Eugene, Oregon, USA) with a sponge-based HydroCel Sensor Net. This system allows full head electrode coverage as it includes electrodes positioned over lower scalp regions and face, which is essential for identification of deep cortical sources, such as those located in orbitofrontal cortex (Luu, Poulsen, \& Tucker, 2009; Luu et al., 2001; Sperli et al., 2006; Tucker, 1993). The sensor net was aligned with respect to three anatomical landmarks; two preauricular points and the nasion. Electrode-to-skin impedances were kept below $50 \mathrm{k} \Omega$ and at equal levels across all electrodes, as recommended for the EGI system we used (Ferree, Luu, Russell, \& Tucker, 2001; Luu, Tucker, Derryberry, Reed, \& Poulsen, 2003; Picton et al., 2000). The recording band-pass filter was $0.001-200 \mathrm{~Hz}$ with sampling rate at $1000 \mathrm{~Hz}$. The electrode $\mathrm{Cz}$ served as the reference.

After application of the EEG cap, participants were led into a dimly lit, sound attenuated room and completed the second gamble task. This task was similar to those used in previous studies (Gehring \& Willoughby, 2002; Hajcak et al., 2006; Nieuwenhuis, Yeung, et al., 2004). Figure 1B shows the flowchart of the trial procedure. Each trial began with a resting interval during which participants viewed a white cross on a black background. Participants then saw two white rectangles positioned next to each other (one on the left and one on the 
right side of the screen). After $1 \mathrm{~s}$, the numbers 25 and 5 were presented in either one of the rectangles. These numbers indicated amount of money (in pence) that could be won or lost on that trial. Each number appeared on either the left or right side of the screen and this was counterbalanced across trials. The rectangles never contained the same number on both sides simultaneously. Participants had to choose between these two options by pressing the left or right mouse button. Their chosen option was highlighted for $1 \mathrm{~s}$ with a yellow rectangle. Next, the chosen and the alternative outcomes were displayed again with the sign of "+" or "" in front of each numeral, indicating their valence. The outcome on any trial was randomly generated by the computer and participants had a $50 \%$ chance of winning or losing. Thus, the prospects could be either positive or negative numbers but participants could not know this in advance. There were four possible combinations of outcomes $(+25+5,+25-5,-25-5,-25$ $+5)$. During the outcome period, participants also received feedback about whether their chosen option was better or worse than the other option. The better of the prospects was highlighted with a green rectangle and the worse prospect with a red rectangle. For example, in the case where both numerals were negative ( -25 vs. -5$)$, participants lost money no matter what they chose. However, losing 5 was still better than losing 25 and, therefore, 5 was highlighted with green. Finally, they were reminded that the value of each chosen outcome would be added to or subtracted from their initial $£ 20$ endowment.

The task consisted of 480 trials, split into 15 blocks of 32 trials. The duration of each block was approximately 5 minutes. At the end of each block, participants received feedback about the amount of money earned in that block as well as the cumulative amount gained from the beginning of the task. 


\subsection{Estimating loss aversion}

We employed a parametric method to estimate the level of loss aversion using a piecewise function:

$$
U(x)=\left\{\begin{array}{ll}
x^{v^{+}}, & x \geq 0 \\
-\lambda(-x)^{v^{-}}, & x<0
\end{array},\right.
$$

where $v$ is the curvature of the value function parameter that controls the diminishing sensitivity, " $x$ " represents the actual outcome from each trail, and $\lambda>1$ is the loss aversion coefficient to overstate disutility from losses. Because the whole utility is reference-dependent, outcomes are regarded as gains when $x \geq 0$ or losses when $x<0$. In line with previous studies (Sokol-Hessner et al., 2009; Tversky and Kahneman, 1992; Wu and Gonzalez, 1996; Benartzi and Thaler, 1995), we employed the assumption of equality of curvature parameters $v^{+}=v^{-}=v$.

The estimation process was based on the logit-function, which gives the probability of acceptance of a risky gamble. Formally, the function can be written as:

$$
F\left(p, x_{g}, x_{l}, x_{c}\right)=\left(1+\exp \left\{-\mu\left(U\left(p, x_{g}, x_{l}\right)-U\left(x_{c}\right)\right)\right\}\right)^{-1}
$$

where $x_{g}$ and $x_{l}$ refer to the monetary amount that participants could win or lose and $x_{c}$ represents the alternative sure outcome. The probability to win the uncertain gamble is represented by $p$. In the present study, we employed the common simplification of linear probability weighting (Tom et al. 2007; Canessa et al. 2013; Schulreich et al. 2016; Sokol-Hessner, 2009, 2013) and probabilities of gains and losses were equal throughout the experiment at $p=(1-\mathrm{p})=0.5$. We further assumed that participants combined their utility and probability in a linear manner, which implies $p U(x)=U(p x)$.

The logit parameter $\mu$ denotes the sensitivity to utility deviations. A greater $\mu$ suggests a greater consistency in applying the respective prospect-theoretic model to individual decision 
making behaviour. On the other hand, smaller $\mu$ indicates more random choice (approaching a random choice with 50:50 probability of acceptance vs. rejection in its extreme).

One hundred choices were collected for each participant. Denote $Z_{i}$ as the choice related to the gamble $i$, where $Z_{i}$ equals one if the participant proceeds with the uncertain gamble, otherwise $Z_{i}$ will remain zero. The log likelihood function is given by:

$$
\sum_{i=1}^{100} Z_{i} \log \left(F\left(p, x_{g}, x_{l}, x_{c}\right)\right)+\left(1-Z_{i}\right) \log \left(1-F\left(p, x_{g}, x_{l}, x_{c}\right)\right)
$$

The values $\lambda, v$ and $\mu$ were obtained by finding a proper set of estimates to maximise Eq. (2). Since this process involved a non-linear optimisation, a numerical approximation method has been applied using the Nelder-Mead simplex algorithm (see Nocedal and Wright (2006)) implemented in Mathematica 9.0 (Wolfram Research, Inc., USA).

\subsection{ERP analysis}

EEG data were pre-processed using BESA v. 6.0 (MEGIS GmbH, Germany). EEG signals were spatially transformed to reference-free data using common average reference method (Lehmann, 1987). This spatial-transformation restored the signal at electrode $\mathrm{Cz}$ which was also used in further analyses. Eye blinks and, when necessary, electrocardiographic artefacts were removed by principal component analysis (Berg \& Scherg, 1994). Further, data were visually inspected for the presence of any movement or muscle artifacts, and epochs contaminated with artifacts were excluded. The average number of accepted trials in each condition was: loss feedback, $215.97 \pm 7.73$ (mean \pm SD); gain feedback, $217.62 \pm 11.10$. The average number of trials accepted did not differ across conditions ( $p>0.05)$. Data were filtered from $0.5-30 \mathrm{~Hz}$. ERPs in response to outcome were computed separately for each condition (gain or loss) by averaging respective epochs in the intervals ranging from $100 \mathrm{~ms}$ 
before outcome onset to $500 \mathrm{~ms}$ after outcome onset. Epochs were baseline corrected using a time window of -100 to 0 ms relative to the onset of feedback.

Data were exported to SPM12 software package (Statistical Parametric Mapping, UCL, England; http://www.fil.ion.ucl.ac.uk/spm/software/spm12). Data from each subject and each outcome condition during the epoch -100 to $500 \mathrm{~ms}$ were converted into three-dimensional scalp-time images. The electrodes were mapped onto a standardised scalp grid sized $32 \times 32$ pixels (pixel size $4.25 \times 5.3 \mathrm{~mm}^{2}$ ) representing the field potential planes stacked over the time axis. Images were smoothed with a Gaussian kernel of $9 \mathrm{~mm} \times 9 \mathrm{~mm} \times 20 \mathrm{~ms}$ (full width at half maximum).

After calculating the contrast of gain-minus-loss, a multivariate regression analysis was computed with the smoothed scalp-time images of 29 participants as the dependent variable, and loss aversion $\lambda$, curvature of the value function $v$ and log-transformed values of choice sensitivity $\mu$ as the predictor variables. The T-contrasts representing positive or negative correlations with $\lambda$ and $v$ were evaluated. An uncorrected $p$ value of 0.001 was used to statistically threshold the data, and significant clusters were only accepted if they were larger than 20 space-time voxels.

\subsection{Source dipole reconstruction}

Grand average potentials comprising both gains and losses, were analysed using source dipole analysis in BESA 6.0 program (MEGIS GmbH, Germany). Equivalent current dipoles (ECDs) were fitted sequentially in the order of peak latencies of individual ERPs evaluated using global field power waveform, similar to previous studies (Hoechstetter et al., 2001; Stancak et al., 2002; Stancak, Ward, \& Fallon, 2013). Classical low-resolution electromagnetic analysis (LORETA; Pascual-Marqui, Michel, \& Lehmann, 1994) recursively applied (CLARA; Hoechstetter, Berg, \& Scherg, 2010) was used as an independent source localisa- 
tion method to verify the presence of each ECD. In iterative steps, CLARA smooths the previous image and sets to zero all voxels with amplitudes of less than $10 \%$ of the maximum activation, effectively eliminating them from the analysis. CLARA analysis employed the singular value decomposition (SVD) regularisation with a cut-off of $0.01 \%$ and four iterations. The source activation images covered the whole brain with a voxel size of $7 \times 7 \times 7 \mathrm{~mm}^{3}$. If a small difference, in the range of $10 \mathrm{~mm}$, in the location of an ECD and a corresponding CLARA cluster was encountered, the fitted ECD maximum was preferred in order to maintain the integrity of the source dipole model over the entire feedback epoch. A 4-shell ellipsoid head volume conductor model was employed, using the following conductivities $(\mathrm{S} / \mathrm{m}=$ Siemens per meter): brain $=0.33 \mathrm{~S} / \mathrm{m}$; scalp $=0.33 \mathrm{~S} / \mathrm{m}$; bone $=0.0042 \mathrm{~S} / \mathrm{m}$; cerebrospinal fluid $(\mathrm{CSF})=1.0 \mathrm{~S} / \mathrm{m}$.

Approximate Talairach coordinates for each ECD were compared with the Talairach atlas (Talairach \& Tournoux, 1988), and the source locations were labelled according to the nearest cortical location.

\section{Results}

\subsection{Choice parameters}

The mean loss aversion $\lambda$ was $1.05 \pm 0.04$ (mean \pm SEM) and the mean curvature of the value function $v$ was $0.53 \pm 0.03$. The mean loss aversion value was slightly smaller compared to previous studies (Sokol-Hessner et al., 2009); however, it fitted well with the mean loss aversion of 1.11 in a recent study involving adolescents and adults (Barkley-Levenson et al., 2013). There was no correlation between loss aversion and curvature of the value function $(p>0.05)$. The assumption of a Gaussian distribution was tested using the Shapiro-Wilk test. Both loss aversion $(W(29)=0.96, p=0.33)$ and curvature of the value function had normal 
distributions $(W(29)=0.94, p=0.12)$. As choice sensitivity $\mu$ was not normally distributed $(p<0.001)$, this variable was log-transformed, resulting in a mean value of $2.31 \pm 0.26$.

\subsection{FRN}

EEG epochs were averaged for each type of outcome (gains and losses), and FRN was quantified by subtracting ERPs to loss trials from ERPs to gain trials (gain-minus-loss difference waveform; Gehring \& Willoughby, 2002). Figure 2A shows grand averaged waveforms of an averaged EEG potential at electrode $\mathrm{Cz}$ at the vertex, and at electrode 38 in the left temporal area for losses and gains. Loss trials $(2.73 \pm 2.14 \mu \mathrm{V})$ resulted in less positive potential amplitudes compared to gain trials $(3.30 \pm 2.29 \mu \mathrm{V} ; t(28)=5.49, p<0.001)$ during the maximum FRN. Figure 2B shows the topographic map of FRN displayed on a volume rendering of a human head. In accordance with previous studies (Gehring \& Willoughby, 2002; Nieuwenhuis, Yeung, et al., 2004), FRN had a positive maximum at central and frontal midline electrodes. However, we also found negative FRN potential components at electrodes overlying the face, and at lower temporal and parietal electrodes. The presence of multiple negative spatial maxima suggests that more than one cortical source contributed to FRN.

\subsection{Loss aversion and FRN}

A multivariate regression analysis was computed involving the three decision making parameters $(\lambda, v$ and $\mu)$ and the smoothed scalp-time maps for the gain-minus-loss contrast in every time sample ranging from -100 to $500 \mathrm{~ms}$ relative to the onset of feedback.

Figure 3A shows the scalp-time plot, a standardised scalp map and a volume rendering of the head representing the statistically significant correlation with loss aversion $\lambda$. One spatio-temporal cluster operating in the interval $233-263 \mathrm{~ms}$ showed a statistically significant negative correlation with $\lambda$ (uncorrected $p<0.001$ ). The temporal maximum of the correla- 
tion between FRN and $\lambda$ had a peak latency of $t=244 \mathrm{~ms}(T=4.64, Z=3.90,547$ scalptime voxels). There were no scalp-time voxels showing any statistically significant positive correlations with $\lambda(p>0.05)$.

To illustrate the correlation between loss aversion and the negative potential during the interval 233-263 ms, the potential value in the scalp-time cluster shown in Figure 3A was evaluated in every subject, and correlated with individual loss aversion values. Figure 3B shows the scatter plot and the linear regression line between $\lambda$ and the cluster representing the negative correlation coefficient of $r(28)=-0.91, p<0.001$.

\subsection{Curvature of the value function and FRN}

To exclude the possibility that the correlation effects of loss aversion overlapped with effects of the curvature of the value function, we also analysed the correlation between the scalp-time images and curvature of the value function. Figure 3D shows the spatio-temporal clusters displaying a statistically significant $(p<0.001)$ positive or negative correlation with curvature of the value function $v$. The statistically significant associations between curvature of the value function and scalp potentials were seen in one scalp-time cluster located in the right frontal region of the scalp and operating in the interval $188-236 \mathrm{~ms}$. The maximum of the correlations between FRN and $v$ had a peak latency of $t=203 \mathrm{~ms}(T=3.75, Z=3.31,76$ scalp-time voxels). Figure 3E shows the scatter plot and the linear regression line between individual curvature of the value function values and the spatio-temporal cluster manifesting a negative correlation $(r(28)=-0.72, p<0.001)$. Therefore, the data showed that correlations of curvature of the value function and scalp-time maps showed a different scalp topographic location and a different latency epoch to those of loss aversion and scalp-time maps. However, the interpretation value of this correlation is limited, owing to the small amplitude of FRN during the 188-236 ms interval (Figure 2A). 


\subsection{Source reconstruction}

Figure 4A shows the grand averaged waveforms and topographic maps of brain activity at different ECDs, on data combined from all the sessions. Figure 4B shows locations of the ECDs, which were fitted using global field power waveform, and spatial clusters obtained in the CLARA analysis. The final source dipole model accounted for $94.3 \%$ of the total variance, and involved five ECDs.

ECD 1 was located in the posterior cingulate gyrus (Brodmann area 31; approximate Talairach coordinates: $x=-4, y=-24, z=45 \mathrm{~mm}$ ) and peaked at $185 \mathrm{~ms}$. ECD 1 had a prevailing radial orientation, related to the positive maximum in the fronto-central electrodes and a negative potential in the lower occipital region of the scalp. ECD 2 was located in the left orbitofrontal cortex (Brodmann area 11; approximate Talairach coordinates: $x=-19, y=3, z=-$ $5 \mathrm{~mm}$ ). This ECD had the negative pole in the left lower facial electrodes and the positive potential pole at parietal electrodes. ECD 2 peaked at $372 \mathrm{~ms}$. ECD 3 was located in the right medial temporal cortex (Brodmann area 35; $x=27, y=0, z=-8 \mathrm{~mm}$ ) and peaked at $388 \mathrm{~ms}$. ECD 3 showed a negative maximum over the occipital electrodes and a positive potential component in the lower frontal region of the scalp. The negative potential over the occipital area of the scalp was located closer to the midline compared to ECD 1, which showed its negative potential component in the right occipital region. ECD 4 was located in the rostral anterior cingulate cortex (Brodmann area 32; approximate Talairach coordinates: $x=-2, y=$ $41, z=8 \mathrm{~mm})$. However, the CLARA cluster also involved the adjacent ventromedial prefrontal cortex (Brodmann area 10), suggesting that ECD 4 picked up activation from both these regions. ECD 4 was a radial dipole showing a strong positive potential pole at the vertex region of the scalp. The earliest peak occurred at $180 \mathrm{~ms}$. ECD 5 was located in the orbitofrontal cortex (Brodmann area 11; $x=6, y=7, z=-2 \mathrm{~mm}$ ). This source showed a doublepeak pattern with peak latencies occurring at $227 \mathrm{~ms}$ and $380 \mathrm{~ms}$. ECD 5 accounted for a 
negative potential component in the chin and neck region and a positive component in the posterior parietal region.

The grand average source dipole model was used to quantify the source waveforms of each of five ECDs in two outcome conditions (loss, gain), and every participant. To test the correlations between loss aversion and feedback related potentials in all five sources over the interval showing the statistically significant correlation with loss aversion (233-263 ms), the mean differences between loss and gain ECD waveforms were calculated in the time epoch of 233-263 ms. Loss aversion values were correlated with five ECDs using the Pearson's correlation method. The only statistically significant correlation coefficient surviving the correction for multiple tests was seen in ECD $5(r(28)=0.38, p<0.05)$. The scatter plot and the linear regression line representing the positive association between the source activity in the orbitofrontal cortex and loss aversion are shown in Figure 3C.

The correlations between curvature of the value function $v$ and five ECDs were computed in the interval showing the statistically significant correlation with $v$ in the scalp potential data (188-236 ms). The only statistically significant correlation coefficient remaining after applying the correction for multiple tests was found in ECD $3(r(28)=0.44, p<0.05)$. The scatter plot and the linear regression line representing the positive association between the source activity in the right medial temporal cortex and curvature of the value function are shown in Figure 3F.

\section{Discussion}

The present study analysed the associations between loss aversion and the spatiotemporal activation patterns during the evaluation of decision outcomes in a monetary gambling task using ERPs. Loss aversion correlated negatively with the amplitude of the negative potential part of FRN in a cluster of electrodes covering the lower face (233-263 ms). The 
correlation between feedback related potentials and loss aversion was featured in the ECD located in the right orbitofrontal cortex. Given that FRN acquired negative signal at the electrodes showing association with loss aversion, the negative correlation corresponds to an increased cortical response to losses in individuals with high levels of loss aversion. The spatiotemporal pattern associated with loss aversion differed from the pattern associated with curvature of the value function; curvature of the value function correlated with FRN in an earlier latency interval (188-236 ms) when FRN was very weak, and the ECD mediating this correlation was located in the right medial temporal cortex.

\subsection{FRN and cortical sources}

FRN potential, evaluated as the difference waveform between loss and gain trials, was consistent with previous studies both in the peak latency, and the fronto-central spatial maximum (Gehring \& Willoughby, 2002; Nieuwenhuis, Yeung, et al., 2004). Our study extends previous research by showing further potential components in the lower facial, temporal, and occipital regions of the scalp, suggesting the presence of more than one dipole. Source localisation of event-related potentials during the outcome period yielded five cortical sources lo-

cated in bilateral OFC, rACC/VMPFC, PCC, and the right medial temporal cortex. This finding accords previous studies reporting the generators of FRN in multiple brain regions (Badgaiyan \& Posner, 1998; Cohen \& Ranganath, 2007; Gehring \& Willoughby, 2002; Hewig et al., 2007; Luu et al., 2003; Miltner et al., 1997; Müller et al., 2005; Nieuwenhuis et al., 2005; Ruchsow et al., 2002; Walsh \& Anderson, 2012).

OFC and VMPFC are prominent parts of the brain valuation system, which is employed in outcome processing (Bartra et al., 2013; Clithero \& Rangel, 2014), in evaluation of goods in the absence of risky decision making (Elliott, Agnew, \& Deakin, 2008; Lebreton et al., 2009) and whilst decisions are made (Chib, Rangel, Shimojo, \& O'Doherty, 2009; Plassmann, 
O'Doherty, \& Rangel, 2010). However, the three additional cortical regions identified in the source dipole model (PCC, rACC and medial temporal cortex) also play roles in decision making.

PCC has been linked to automatic subjective value computation (Grueschow, Polania, Hare, \& Ruff, 2015), comparison between alternative choices (FitzGerald, Seymour, \& Dolan, 2009) and reward magnitude (Ballard \& Knutson, 2009). Additionally, the peak latency of the source located in PCC corresponded to the P200 component, which has been shown to encode the predictability of outcomes (Polezzi, Lotto, Daum, Sartori, \& Rumiati, 2008), magnitude of monetary outcomes (San Martín, Appelbaum, Pearson, Huettel, \& Woldorff, 2013) and outcome history (Osinsky, Mussel, \& Hewig, 2012).

Activations in rACC have been associated with emotional processing (Bush, Luu, \& Posner, 2000), error detection (Kiehl, Liddle, \& Hopfinger, 2000; Menon, Adleman, White, Glover, \& Reiss, 2001; Rubia, Smith, Brammer, \& Taylor, 2003; Ullsperger \& von Cramon, 2001) and coding of reward value (Di Pellegrino, Ciaramelli, \& Làdavas, 2007; Marsh, Blair, Vythilingam, Busis, \& Blair, 2007).

As far as the source dipole in medial temporal cortex is concerned, previous studies reported activations associated with negative feedback (Coricelli et al., 2005), reward expectancies (Ramnani, Elliott, Athwal, \& Passingham, 2004) and risk taking (Paulus et al., 2001).

Overall, our results show that processing the outcomes of decisions in a monetary gamble task involves activations of brain regions implicated in assigning values to goods, emotions, reward and punishment, and monitoring outcomes and errors.

\subsection{Loss aversion and FRN}

Loss aversion modulated the amplitude of FRN in the early latency period of 233-263 ms on the ascending limb of FRN peak (275 ms). Due to rigorous statistical thresholding, 
which was necessary to account for multiple tests, only one small space-time cluster of activation has survived the correction. However, this cluster was part of a strong negative FRN component seen at the whole left lower face (Figure 2). The modulation of FRN in lower facial electrodes suggests that one or more deep cortical sources were involved (Luu et al., 2009; Luu et al., 2001; Sperli et al., 2006; Tucker, 1993). Indeed, the negative potential component seen at the face was associated with the ECD in the right OFC, which is where the correlation with loss aversion was found. OFC has been reported to be involved in computing the values of goods (Elliott et al., 2008; Lebreton et al., 2009), encoding reward/punishment magnitude (O'Doherty, Kringelbach, Rolls, Hornak, \& Andrews, 2001; Roesch \& Olson, 2004; Tremblay \& Schultz, 1999) and mediating hedonic experience and evaluation of affective valence of stimuli (Cunningham, Kesek, \& Mowrer, 2009; Kringelbach, O’Doherty, Rolls, \& Andrews, 2003). Given the importance of OFC in hedonic evaluation of decision outcomes and the specific relation of loss aversion to outcomes of negative hedonic value, the present data are consistent with the role of OFC in decision making.

Previous studies have shown that processing of positive emotional stimuli are associated with activity in the left hemisphere, whereas processing of negative emotional stimuli are associated with activity in the right hemisphere (Ahern \& Schwartz, 1985; Canli, Desmond, Zhao, Glover, \& Gabrieli, 1998; Davidson, 1998; Lane et al., 1997; Lang et al., 1998; Mandal, Tandon, \& Asthana, 1991; Tucker, 1981; Windmann et al., 2006). Although the outcome period was associated with activation in bilateral OFC, only the right OFC showed a statistically significant correlation with loss aversion. Given that loss aversion is a response to a negative prospect/outcome (monetary loss), this right-hemisphere lateralisation in the correlation between OFC and loss aversion is in line with the right-hemisphere prevalence in perception of hedonically negative stimuli (Ahern \& Schwartz, 1985; Canli et al., 1998; 
Davidson, 1998; Lane et al., 1997; Lang et al., 1998; Mandal et al., 1991; Tucker, 1981; Windmann et al., 2006).

The present study adds to previous data showing that individual levels of loss aversion correlated with activations in the VMPFC (Tom et al., 2007), ACC (Canessa et al., 2013), and ventral striatum (Canessa et al., 2013; Tom et al., 2007) during the decision period, and in amygdala during the outcome period (Sokol-Hessner et al., 2013). Our data suggests that OFC provides an individually tuned neural signal about subjective value of loss or gain, and that this signal is associated with the tendency to avoid losses manifested in declining monetary gambles. Further studies should address whether the correlation between the activation in OFC and loss aversion during the outcome period would be also found in event-related potentials during the decision period.

Although we also found a correlation between the curvature of the value function and the scalp-time maps, the correlation between ERPs and the curvature of the value function in the interval 188-236 ms was not interpreted due to the weak FRN signal in this latency interval. However, the spatial location of the curvature of the value function correlation cluster, the time epoch, and the cortical source displaying a correlation with curvature of the value function differed from loss aversion data. These differences, together with the lack of correlation between loss aversion and curvature of the value function, suggest that the correlation between loss aversion and FRN seen in the present study was not contaminated with curvature of the value function.

To conclude, the individual level of loss aversion is associated with the strength of electrocortical response to decision outcomes. Results suggest that increased neural signals for loss outcomes in the orbitofrontal cortex are associated with utility functions that are steeper for losses than gains during decision making under risk. Although the present study shows an association between loss aversion and activation in OFC only during the evaluation of deci- 
sion outcomes, it is possible that a similar mechanism is also implemented during the evaluation of anticipated outcomes in the course of the decision phase.

\section{Role of the funding source}

Dr. N. Fallon's post-doctoral fellowship was sponsored by Unilever. Miss K. Kokmotou was supported by the Centre for Doctoral Training, funded by the ESRC and EPSRC (United Kingdom). This work was approved for publication by Unilever's Research and Development department. 
A
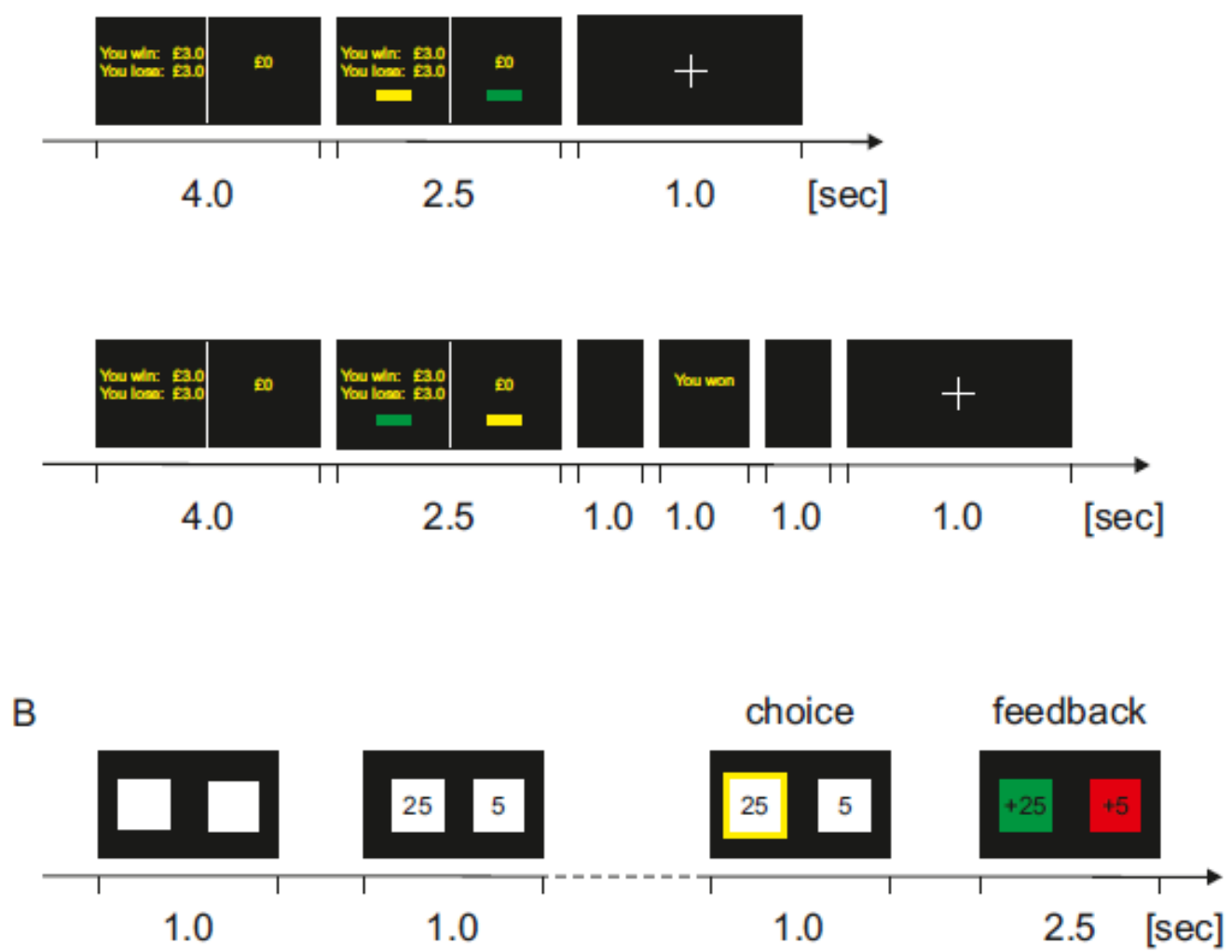

Figure 1 A. Trial structure of loss aversion task. Top panel: Declined gambles. Each trial began with a fixation cross, followed by the presentation of two possible choices, which were displayed on the screen for $4 \mathrm{~s}$. Half of the screen presented a gamble option (e.g. "you win $£ 3.0$, you lose $£ 3.0$ ”) with a $50 \%$ chance of winning or losing the displayed amount of money. The other half of the screen showed the value of a sure outcome. Participants were instructed to choose between the two prospects by pressing the left or the right mouse button according to the part of the screen they preferred. If participants chose a sure zero outcome, they would neither lose nor win anything. In the next $2.5 \mathrm{~s}$, the options stayed on the screen and two yellow rectangles appeared at the bottom of the screen. Subsequently, a fixation cross appeared on the screen and the next trial started after $1 \mathrm{~s}$. Bottom panel: Accepted gambles. If participants selected the risky gamble option, a black screen was displayed for $1 \mathrm{~s}$ af- 
ter the $2.5 \mathrm{~s}$ response period, and feedback about the gamble outcome was shown for $1 \mathrm{~s}$ ("You won" or "You lost"). A 1 s black screen served as a resting period before the next trial. B. Trial structure in the FRN task. Each trial began with the display of two white rectangles positioned next to each other (one on the left and one on the right side of the screen) on a black background. After $1 \mathrm{~s}$, the numerals 25 and 5 were presented in either one of the rectangles. These numbers indicated amount of money (in pence). Participants had to choose between these two options by pressing the left or right mouse button. Their chosen option was highlighted for $1 \mathrm{~s}$ with a yellow rectangle. After this, the chosen and the alternative outcomes were displayed with the sign + or - in front of each numeral, indicating their valence. In addition, participants received feedback about whether their chosen option was better or worse than the unchosen one. The best prospect was highlighted with green colour and the worst with red colour. 
A
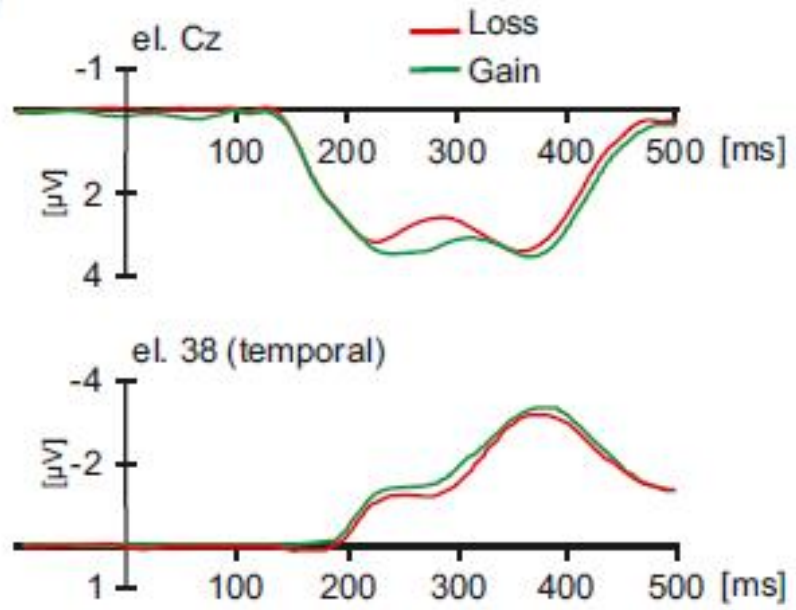

B
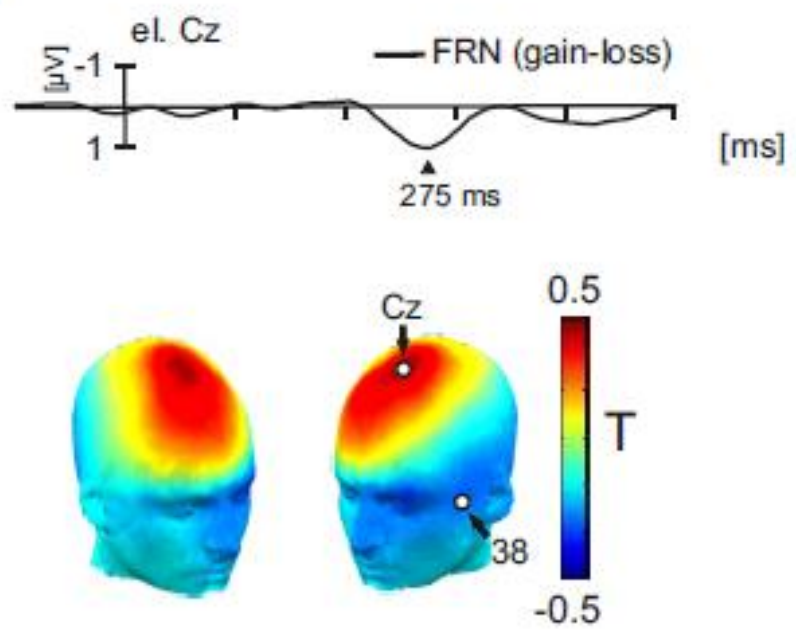

Figure 2 A. Grand averaged EEG potentials for gain and loss trials shown at electrode $\mathrm{Cz}$ at the vertex, and at electrode 38 in the left temporal area of the scalp. B. FRN is shown as the amplitude difference between gain and loss trials, peaking at $275 \mathrm{~ms}$ after feedback presentation (top panel). The scalp topographic map of FRN at its peak (275 ms) latency (bottom panel). 
A

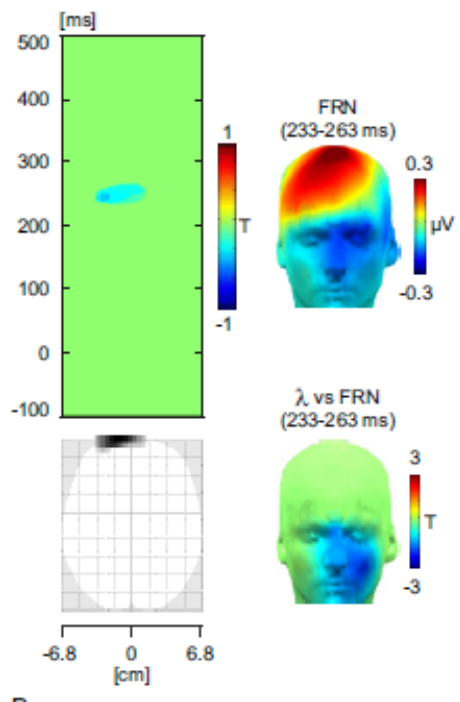

B

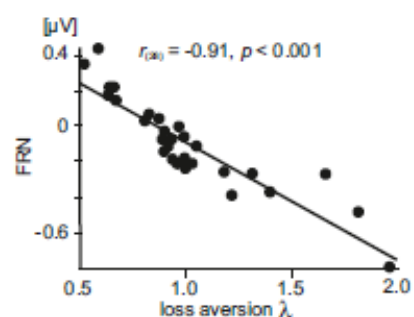

C

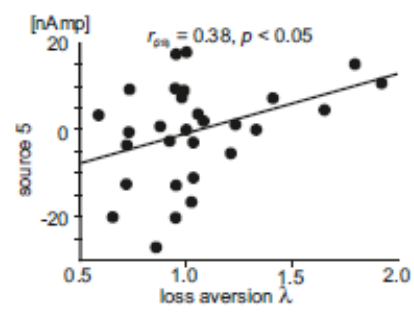

D

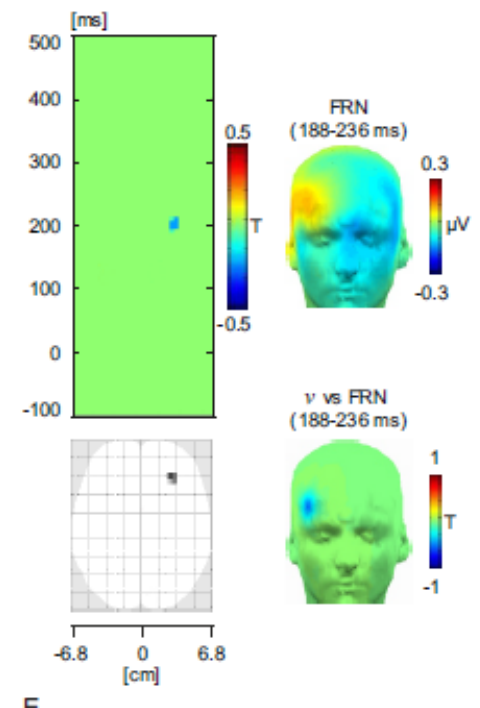

E

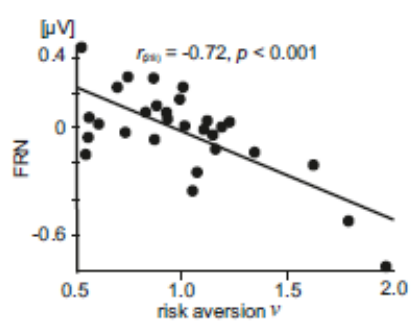

$\mathrm{F}$

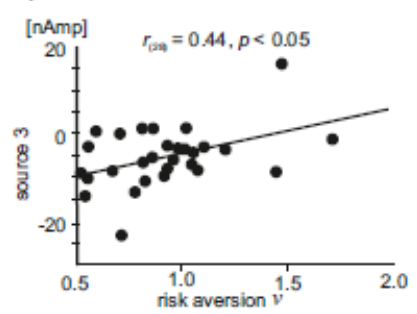

Figure 3 Correlations between FRN and loss aversion and curvature of the value function. A. The vertical green scalp-time plot shows one statistically significant regression between FRN and loss aversion (uncorrected $p<0.001$ ). The $\mathrm{T}$ values represent the strength and direction of regression over the horizontal axis of the scalp in every time sample from $-100 \mathrm{~ms}$ to 500 ms. The scalp values over the horizontal axis of the scalp are averages of $\mathrm{T}$ values occurring at each vertical point in time for a given horizontal point in the standardised scalp map (from $-6.8 \mathrm{~cm}$ to $+6.8 \mathrm{~cm})$. One interval showed the presence of a statistically significant spatiotemporal cluster. In the interval $233-263 \mathrm{~ms}$, one cluster showed a statistically significant negative correlation between loss aversion and FRN. Below the green panel is the standard 
scalp map of statistically significant negative regression between loss aversion and FRN. The horizontal axis of the standardised scalp-time map is aligned with the space-time map above. In the right part of this panel, there are two topographic maps. The upper map shows the FRN potential, and the lower map shows the topographic map of the statistically significant regression between loss aversion and FRN in T values. B. The scatter plot and linear regression line representing the correlation between loss aversion and the strength of FRN, $r(28)=-0.91, p<$ 0.001. C. The scatter plot and linear regression line demonstrating the correlation between loss aversion scores and the strength of ECD 5 located in the right OFC, $\mathrm{r}(28)=0.38, \mathrm{p}<$ 0.05. D. The scalp-time plot of the regression between curvature of the value function and scalp-time maps. In the interval $188-236 \mathrm{~ms}$, one cluster showed a statistically significant negative correlation between curvature of the value function and FRN. The scalp map below the scalp-time plot is the standardised topographic map and shows the topographic location of the cluster showing the statistically significant correlation with curvature of the value function. The two topographic maps in the right part of this panel are the FRN potential map at $t=$ 188-236 ms, and the regression map representing the associations between FRN and curvature of the value function at $t=188-236 \mathrm{~ms}$. E. The scatter plot and linear regression representing the association between curvature of the value function and FRN, r(28) $=-0.72, p<$ 0.001. F. The correlation between curvature of the value function and source dipole moments in $\operatorname{ECD} 3, \mathrm{r}(28)=0.44, \mathrm{p}<0.05$. 


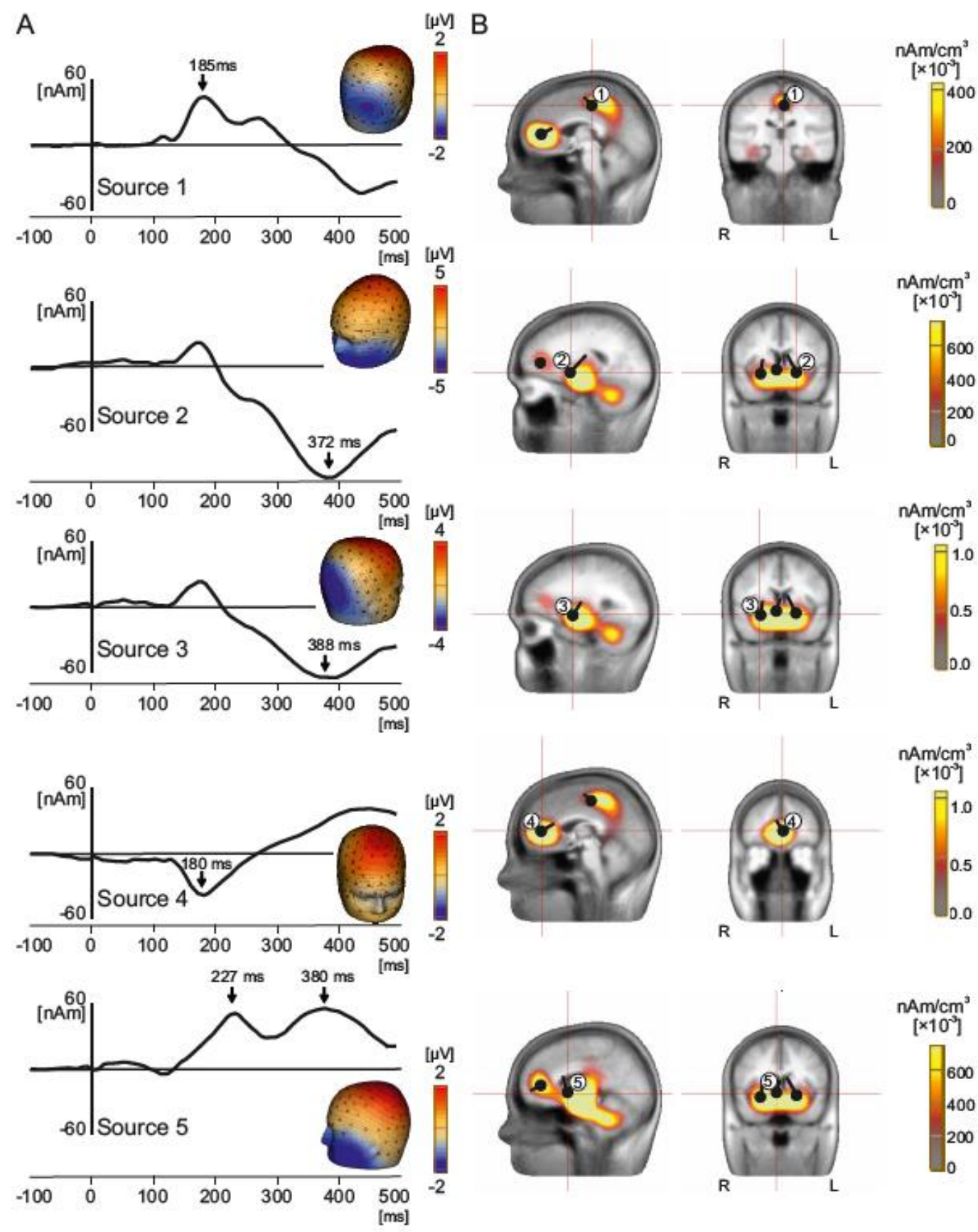

Figure 4 Source dipole model and source waveforms underlying event-related potentials during the outcome period. A. Grand average source waveforms and the topographic scalp maps in five ECDs. Peak latencies are highlighted with arrows. B. Locations of five ECDs in a standard 3-D anatomical MR image and respective CLARA cluster (yellow-orange). Each source is represented by a bar seeded using global field power waveform. The ECDs are associated with numbers, which correspond to the source numbers in $(\mathrm{A}) . \mathrm{L}=$ left, $\mathrm{R}=$ right. 


\section{References}

Ahern, G. L., \& Schwartz, G. E. (1985). Differential lateralization for positive and negative emotion in the human brain: EEG spectral analysis. Neuropsychologia, 23, 745-755.

Badgaiyan, R. D., \& Posner, M. I. (1998). Mapping the cingulate cortex in response selection and monitoring. NeuroImage, 7, 255-260.

Ballard, K., \& Knutson, B. (2009). Dissociable neural representations of future reward magnitude and delay during temporal discounting. NeuroImage, 45, 143-150.

Barkley-Levenson, E. E., Van Leijenhorst, L., \& Galván, A. (2013). Behavioral and neural correlates of loss aversion and risk avoidance in adolescents and adults. Developmental Cognitive Neuroscience, 3, 72-83.

Bartra, O., McGuire, J. T., \& Kable, J. W. (2013). The valuation system: a coordinate-based meta-analysis of BOLD fMRI experiments examining neural correlates of subjective value. NeuroImage, 76, 412-427.

Bellebaum, C., \& Daum, I. (2008). Learning-related changes in reward expectancy are reflected in the feedback-related negativity. European Journal of Neuroscience, 27, 1823-1835.

Berg, P., \& Scherg, M. (1994). A multiple source approach to the correction of eye artifacts. Electroencephalography and Clinical Neurophysiology, 90, 229-241.

Bush, G., Luu, P., \& Posner, M. I. (2000). Cognitive and emotional influences in anterior cingulate cortex. Trends in Cognitive Sciences, 4, 215-222.

Canessa, N., Crespi, C., Motterlini, M., Baud-Bovy, G., Chierchia, G., Pantaleo, G., et al. (2013). The functional and structural neural basis of individual differences in loss aversion. Journal of Neuroscience, 33, 14307-14317.

Canli, T., Desmond, J. E., Zhao, Z., Glover, G., \& Gabrieli, J. D. E. (1998). Hemispheric asymmetry for emotional stimuli detected with fMRI. Neuroreport, 9, 3233-3239. 
Chib, V. S., Rangel, A., Shimojo, S., \& O'Doherty, J. P. (2009). Evidence for a common representation of decision values for dissimilar goods in human ventromedial prefrontal cortex. Journal of Neuroscience, 29, 12315-12320.

Clithero, J. A., \& Rangel, A. (2014). Informatic parcellation of the network involved in the computation of subjective value. Social Cognitive and Affective Neuroscience, 9, 1289-1302.

Cohen, M. X., \& Ranganath, C. (2007). Reinforcement learning signals predict future decisions. Journal of Neuroscience, 27, 371-378.

Coricelli, G., Critchley, H. D., Joffily, M., O'Doherty, J. P., Sirigu, A., \& Dolan, R. J. (2005). Regret and its avoidance: a neuroimaging study of choice behavior. Nature neuroscience, $8,1255-1262$.

Cunningham, W. A., Kesek, A., \& Mowrer, S. M. (2009). Distinct orbitofrontal regions encode stimulus and choice valuation. Journal of Cognitive Neuroscience, 21, 19561966.

Davidson, R. J. (1998). Affective style and affective disorders: Perspectives from affective neuroscience. Cognition \& Emotion, 12, 307-330.

Delgado, M. R., Nystrom, L. E., Fissell, C., Noll, D. C., \& Fiez, J. A. (2000). Tracking the hemodynamic responses to reward and punishment in the striatum. Journal of Neurophysiology, 84, 3072-3077.

Di Pellegrino, G., Ciaramelli, E., \& Làdavas, E. (2007). The regulation of cognitive control following rostral anterior cingulate cortex lesion in humans. Journal of Cognitive Neuroscience, 19, 275-286.

Elliott, R., Agnew, Z., \& Deakin, J. F. W. (2008). Medial orbitofrontal cortex codes relative rather than absolute value of financial rewards in humans. European Journal of Neuroscience, 27, 2213-2218. 
Ferree, T. C., Luu, P., Russell, G. S., \& Tucker, D. M. (2001). Scalp electrode impedance, infection risk, and EEG data quality. Clinical Neurophysiology, 112, 536-544.

FitzGerald, T. H. B., Seymour, B., \& Dolan, R. J. (2009). The role of human orbitofrontal cortex in value comparison for incommensurable objects. Journal of Neuroscience, $29,8388-8395$.

Gehring, W. J., \& Willoughby, A. R. (2002). The medial frontal cortex and the rapid processing of monetary gains and losses. Science, 295, 2279-2282.

Glöckner, A., \& Pachur, T. (2012). Cognitive models of risky choice: Parameter stability and predictive accuracy of prospect theory. Cognition, 123, 21-32.

Grueschow, M., Polania, R., Hare, T. A., \& Ruff, C. C. (2015). Automatic versus choicedependent value representations in the human brain. Neuron, 85, 874-885.

Hajcak, G., Moser, J. S., Holroyd, C. B., \& Simons, R. F. (2006). The feedback-related negativity reflects the binary evaluation of good versus bad outcomes. Biological Psychology, 71, 148-154.

Hardie, B. G. S., Johnson, E. J., \& Fader, P. S. (1993). Modeling loss aversion and reference dependence effects on brand choice. Marketing Science, 12, 378-394.

Hauser, T. U., Iannaccone, R., Stämpfli, P., Drechsler, R., Brandeis, D., Walitza, S., et al. (2014). The feedback-related negativity (FRN) revisited: new insights into the localization, meaning and network organization. NeuroImage, 84, 159-168.

Heeren, G., Markett, S., Montag, C., Gibbons, H., \& Reuter, M. (2016). Decision conflict and loss aversion-An ERP study. Journal of Neuroscience, Psychology, and Economics, $9,50$.

Hewig, J., Trippe, R., Hecht, H., Coles, M. G. H., Holroyd, C. B., \& Miltner, W. H. R. (2007). Decision-making in Blackjack: an electrophysiological analysis. Cerebral Cortex, 17, 865-877. 
Hoechstetter, K., Berg, P., \& Scherg, M. (2010). BESA research tutorial 4: Distributed source imaging. BESA Research Tutorial, 1-29.

Hoechstetter, K., Rupp, A., Stančák, A. j., Meinck, H. M., Stippich, C., Berg, P., et al. (2001). Interaction of tactile input in the human primary and secondary somatosensory cortex - a magnetoencephalographic study. NeuroImage, 14, 759-767.

Kahneman, D., Knetsch, J. L., \& Thaler, R. H. (1990). Experimental tests of the endowment effect and the Coase theorem. Journal of political Economy, 1325-1348.

Kahneman, D., \& Tversky, A. (1979). Prospect theory: An analysis of decision under risk. Econometrica: Journal of the econometric society, 263-291.

Kiehl, K. A., Liddle, P. F., \& Hopfinger, J. B. (2000). Error processing and the rostral anterior cingulate: An event-related fMRI study. Psychophysiology, 37, 216-223.

Knutson, B., Adams, C. M., Fong, G. W., \& Hommer, D. (2001). Anticipation of increasing monetary reward selectively recruits nucleus accumbens. Journal of Neuroscience, 21, RC159.

Kringelbach, M. L., O’Doherty, J., Rolls, E. T., \& Andrews, C. (2003). Activation of the human orbitofrontal cortex to a liquid food stimulus is correlated with its subjective pleasantness. Cerebral Cortex, 13, 1064-1071.

Lane, R. D., Reiman, E. M., Bradley, M. M., Lang, P. J., Ahern, G. L., Davidson, R. J., et al. (1997). Neuroanatomical correlates of pleasant and unpleasant emotion. Neuropsychologia, 35, 1437-1444.

Lang, P. J., Bradley, M. M., Fitzsimmons, J. R., Cuthbert, B. N., Scott, J. D., Moulder, B., et al. (1998). Emotional arousal and activation of the visual cortex: an fMRI analysis. Psychophysiology, 35, 199-210. 
Lebreton, M., Jorge, S., Michel, V., Thirion, B., \& Pessiglione, M. (2009). An automatic valuation system in the human brain: evidence from functional neuroimaging. Neuron, 64, 431-439.

Lehmann, D. (1987). Principles of spatial analysis. Handbook of electroencephalography and clinical neurophysiology, 1, 309-354.

Luu, P., Poulsen, C., \& Tucker, D. M. (2009). Neurophysiological measures of brain activity: Going from the scalp to the brain. Paper presented at the International Conference on Foundations of Augmented Cognition.

Luu, P., Tucker, D. M., Derryberry, D., Reed, M., \& Poulsen, C. (2003). Electrophysiological responses to errors and feedback in the process of action regulation. Psychological Science, 14, 47-53.

Luu, P., Tucker, D. M., Englander, R., Lockfeld, A., Lutsep, H., \& Oken, B. (2001). Localizing Acute Stroke-related EEG Changes:: Assessing the Effects of Spatial Undersampling. Journal of Clinical Neurophysiology, 18, 302-317.

Ma, Q., Feng, Y., Xu, Q., Bian, J., \& Tang, H. (2012). Brain potentials associated with the outcome processing in framing effects. Neuroscience Letters, 528, 110-113.

Mandal, M. K., Tandon, S. C., \& Asthana, H. S. (1991). Right brain damage impairs recognition of negative emotions. Cortex, 27, 247-253.

Marsh, A. A., Blair, K. S., Vythilingam, M., Busis, S., \& Blair, R. J. R. (2007). Response options and expectations of reward in decision-making: the differential roles of dorsal and rostral anterior cingulate cortex. Neurolmage, 35, 979-988.

Martin, L. E., Potts, G. F., Burton, P. C., \& Montague, P. R. (2009). Electrophysiological and hemodynamic responses to reward prediction violation. Neuroreport, 20, 1140. 
May, J. C., Delgado, M. R., Dahl, R. E., Stenger, V. A., Ryan, N. D., Fiez, J. A., et al. (2004). Event-related functional magnetic resonance imaging of reward-related brain circuitry in children and adolescents. Biological Psychiatry, 55, 359-366.

Menon, V., Adleman, N. E., White, C. D., Glover, G. H., \& Reiss, A. L. (2001). Error-related brain activation during a Go/NoGo response inhibition task. Human brain mapping, $12,131-143$.

Miltner, W. H. R., Braun, C. H., \& Coles, M. G. H. (1997). Event-related brain potentials following incorrect feedback in a time-estimation task: Evidence for a "generic" neural system for error detection. Journal of Cognitive Neuroscience, 9, 788-798.

Müller, S. V., Möller, J., Rodriguez-Fornells, A., \& Münte, T. F. (2005). Brain potentials related to self-generated and external information used for performance monitoring. Clinical Neurophysiology, 116, 63-74.

Nieuwenhuis, S., Holroyd, C. B., Mol, N., \& Coles, M. G. H. (2004). Reinforcement-related brain potentials from medial frontal cortex: origins and functional significance. Neuroscience \& Biobehavioral Reviews, 28, 441-448.

Nieuwenhuis, S., Slagter, H. A., Geusau, V., Alting, N. J., Heslenfeld, D. J., \& Holroyd, C. B. (2005). Knowing good from bad: differential activation of human cortical areas by positive and negative outcomes. European Journal of Neuroscience, 21, 3161-3168.

Nieuwenhuis, S., Yeung, N., Holroyd, C. B., Schurger, A., \& Cohen, J. D. (2004). Sensitivity of electrophysiological activity from medial frontal cortex to utilitarian and performance feedback. Cerebral Cortex, 14, 741-747.

Nocedal, J., \& Wright, S. (2006). Numerical optimization: Springer Science \& Business Media. 
O'Doherty, J., Kringelbach, M. L., Rolls, E. T., Hornak, J., \& Andrews, C. (2001). Abstract reward and punishment representations in the human orbitofrontal cortex. Nature neuroscience, 4, 95-102.

Osinsky, R., Mussel, P., \& Hewig, J. (2012). Feedback-related potentials are sensitive to sequential order of decision outcomes in a gambling task. Psychophysiology, 49, 1579-1589.

Pascual-Marqui, R. D., Michel, C. M., \& Lehmann, D. (1994). Low resolution electromagnetic tomography: a new method for localizing electrical activity in the brain. International Journal of Psychophysiology, 18, 49-65.

Paulus, M. P., Hozack, N., Zauscher, B., McDowell, J. E., Frank, L., Brown, G. G., et al. (2001). Prefrontal, parietal, and temporal cortex networks underlie decision-making in the presence of uncertainty. NeuroImage, 13, 91-100.

Picton, T. W., Bentin, S., Berg, P., Donchin, E., Hillyard, S. A., Johnson, R., et al. (2000). Guidelines for using human event-related potentials to study cognition: recording standards and publication criteria. Psychophysiology, 37, 127-152.

Plassmann, H., O'Doherty, J. P., \& Rangel, A. (2010). Appetitive and aversive goal values are encoded in the medial orbitofrontal cortex at the time of decision making. Journal of Neuroscience, 30, 10799-10808.

Polezzi, D., Lotto, L., Daum, I., Sartori, G., \& Rumiati, R. (2008). Predicting outcomes of decisions in the brain. Behavioural brain research, 187, 116-122.

Pope, D. G., \& Schweitzer, M. E. (2011). Is Tiger Woods loss averse? Persistent bias in the face of experience, competition, and high stakes. The American Economic Review, $101,129-157$. 
Potts, G. F., Martin, L. E., Burton, P. C., \& Montague, P. R. (2006). When things are better or worse than expected: the medial frontal cortex and the allocation of processing resources. Journal of Cognitive Neuroscience, 18, 1112-1119.

Putler, D. S. (1992). Incorporating reference price effects into a theory of consumer choice. Marketing Science, 11, 287-309.

Ramnani, N., Elliott, R., Athwal, B. S., \& Passingham, R. E. (2004). Prediction error for free monetary reward in the human prefrontal cortex. NeuroImage, 23, 777-786.

Roesch, M. R., \& Olson, C. R. (2004). Neuronal activity related to reward value and motivation in primate frontal cortex. Science, 304, 307-310.

Rubia, K., Smith, A. B., Brammer, M. J., \& Taylor, E. (2003). Right inferior prefrontal cortex mediates response inhibition while mesial prefrontal cortex is responsible for error detection. NeuroImage, 20, 351-358.

Ruchsow, M., Grothe, J., Spitzer, M., \& Kiefer, M. (2002). Human anterior cingulate cortex is activated by negative feedback: evidence from event-related potentials in a guessing task. Neuroscience Letters, 325, 203-206.

San Martín, R., Appelbaum, L. G., Pearson, J. M., Huettel, S. A., \& Woldorff, M. G. (2013). Rapid brain responses independently predict gain maximization and loss minimization during economic decision making. Journal of Neuroscience, 33, 7011-7019.

Santesso, D. L., Dzyundzyak, A., \& Segalowitz, S. J. (2011). Age, sex and individual differences in punishment sensitivity: Factors influencing the feedback-related negativity. Psychophysiology, 48, 1481-1489.

Schulreich, S., Gerhardt, H., \& Heekeren, H. R. (2016). Incidental fear cues increase monetary loss aversion. Emotion, 16, 402. 
Sokol-Hessner, P., Camerer, C. F., \& Phelps, E. A. (2013). Emotion regulation reduces loss aversion and decreases amygdala responses to losses. Social Cognitive and Affective Neuroscience, nss002.

Sokol-Hessner, P., Hsu, M., Curley, N. G., Delgado, M. R., Camerer, C. F., \& Phelps, E. A. (2009). Thinking like a trader selectively reduces individuals' loss aversion. Proceedings of the National Academy of Sciences, 106, 5035-5040.

Sperli, F., Spinelli, L., Seeck, M., Kurian, M., Michel, C. M., \& Lantz, G. (2006). EEG source imaging in pediatric epilepsy surgery: a new perspective in presurgical workup. Epilepsia, 47, 981-990.

Stancak, A., Hoechstetter, K., Tintera, J., Vrana, J., Rachmanova, R., Kralik, J., et al. (2002). Source activity in the human secondary somatosensory cortex depends on the size of corpus callosum. Brain research, 936, 47-57.

Stancak, A., Ward, H., \& Fallon, N. (2013). Modulation of pain by emotional sounds: a laser-evoked potential study. European Journal of Pain, 17, 324-335.

Stancak, A., Xie, Y., Fallon, N., Bulsing, P., Giesbrecht, T., Thomas, A., et al. (2015). Unpleasant odors increase aversion to monetary losses. Biological Psychology, 107, $1-9$.

Takahashi, H., Fujie, S., Camerer, C. F., Arakawa, R., Takano, H., Kodaka, F., et al. (2012). Norepinephrine in the brain is associated with aversion to financial loss. Molecular Psychiatry, 18, 3-4.

Talairach, J., \& Tournoux, P. (1988). Co-planar stereotaxic atlas of the human brain. 3Dimensional proportional system: an approach to cerebral imaging.

Tom, S. M., Fox, C. R., Trepel, C., \& Poldrack, R. A. (2007). The neural basis of loss aversion in decision-making under risk. Science, 315, 515-518. 
Tremblay, L., \& Schultz, W. (1999). Relative reward preference in primate orbitofrontal cortex. Nature, 398, 704-708.

Tucker, D. M. (1981). Lateral brain function, emotion, and conceptualization. Psychological bulletin, 89, 19.

Tucker, D. M. (1993). Spatial sampling of head electrical fields: the geodesic sensor net. Electroencephalography and clinical neurophysiology, 87, 154-163.

Ullsperger, M., \& von Cramon, D. Y. (2001). Subprocesses of performance monitoring: a dissociation of error processing and response competition revealed by event-related fMRI and ERPs. NeuroImage, 14, 1387-1401.

Unger, K., Heintz, S., \& Kray, J. (2012). Punishment sensitivity modulates the processing of negative feedback but not error-induced learning. Frontiers in human neuroscience, 6.

Walsh, M. M., \& Anderson, J. R. (2012). Learning from experience: Event-related potential correlates of reward processing, neural adaptation, and behavioral choice. Neuroscience \& Biobehavioral Reviews, 36, 1870-1884.

Windmann, S., Kirsch, P., Mier, D., Stark, R., Walter, B., Güntürkün, O., et al. (2006). On framing effects in decision making: linking lateral versus medial orbitofrontal cortex activation to choice outcome processing. Journal of Cognitive Neuroscience, 18, 1198-1211.

Wright, N. D., Symmonds, M., Hodgson, K., Fitzgerald, T. H. B., Crawford, B., \& Dolan, R. J. (2012). Approach-avoidance processes contribute to dissociable impacts of risk and loss on choice. Journal of Neuroscience, 32, 7009-7020.

Yeung, N., Holroyd, C. B., \& Cohen, J. D. (2005). ERP correlates of feedback and reward processing in the presence and absence of response choice. Cerebral cortex, 15, 535544. 
Yeung, N., \& Sanfey, A. G. (2004). Independent coding of reward magnitude and valence in the human brain. The Journal of Neuroscience, 24, 6258-6264.

Yu, R., \& Zhang, P. (2014). Neural evidence for description dependent reward processing in the framing effect. Frontiers in neuroscience, 8. 